\title{
Modelagem de Conceitos e Processos Matemáticos por Redes de Petri Coloridas: o caso da integrabilidade de funções reais
}

\section{Modeling of Concepts and Mathematical Processes by Petri Nets: the case of integrability of real functions}

\author{
Natália Maria Cordeiro Barroso* \\ José Marques Soares** \\ Giovanni Cordeiro Barroso**** \\ João Cesar Moura Mota**** \\ Hermínio Borges Neto*****
}

\begin{abstract}
Resumo
As redes de Petri (RPs) constituem-se em uma ferramenta matemática e gráfica de uso geral. Sugere-se, neste trabalho, o uso de RPs como uma ferramenta de modelagem

\footnotetext{
* Doutora em Engenharia de Teleinformática pela Universidade Federal do Ceará (UFC). Professora Adjunta do Centro de Tecnologia da Universidade Federal do Ceará (UFC), Fortaleza, CE, Brasil. Endereço para correspondência: Campus do Pici, s/n, Bloco 710, CEP: 60455-760, Fortaleza, CE, Brasil.E-mail: natalia@ufc.br.

${ }^{* *}$ Doutor em Réseaux, Connaissances et Organisations pelo Institut National de Télécommunications (INT), Évry, França. Professor Adjunto do Departamento de Engenharia de Teleinformática da Universidade Federal do Ceará (UFC), Fortaleza, CE, Brasil. Endereço para correspondência: Campus do Pici, s/n, Bloco 725, CEP 60455-970, Fortaleza, CE, Brasil. E-mail: marques@ ufc.br.

*** Doutor em Engenharia Elétrica pela Universidade Federal da Paraíba (UFPB). Professor Associado do Departamento de Física da Universidade Federal do Ceará (UFC). Endereço para correspondência: Campus do Pici, Bloco 928, sala 39, CEP: 60455-970, Fortaleza, CE, Brasil. E-mail: gcb@ fisica.ufc.br.

***** Doutor em Engenharia Elétrica pela Universidade Estadual de Campinas (UNICAMP). Professor Titular do Departamento de Engenharia de Teleinformática da Universidade Federal do Ceará (UFC), Fortaleza, CE, Brasil. Endereço para correspondência: Campus do Pici, s/n, Bloco 725, CEP 60455-970, Fortaleza, CE, Brasil.E-mail: mota@gtel.ufc.br.

****** Doutor em Matemática pelo Instituto de Matemática Pura e Aplicada (IMPA). Professor Associado da Faculdade de Educação (FACED) da Universidade Federal do Ceará (UFC), Fortaleza, CE, Brasil. Endereço para correspondência: Rua Waldery Uchoa, 1, Benfica, CEP: 60020-110, Fortaleza, CE, Brasil. E-mail: herminio@ufc.br.
} 
voltada para o ensino de noções matemáticas. A modelagem apoia-se no fato de as RPs terem como característica a representação de sistemas que utilizam forte base matemática. Como estudo de caso, apresenta-se uma modelagem para a organização do conteúdo de ensino sobre integrabilidade de funções reais baseada na evolução do conceito de integral. Para isto, considera-se uma função limitada, definida em um intervalo fechado e limitado da reta, que é analisada relativamente a condições que garantam ou excluam a sua integrabilidade por Cauchy, por Riemann, por Lebesgue ou por nenhum destes. A ideia de se modelar este conceito por RPs originou-se no contexto da sala de aula, por ocasião de seu ensino a alunos de primeiro ano de engenharia.

Palavras-chave: Modelagem. Ensino. Redes de Petri. Integral

\begin{abstract}
Petri Nets (PNs) are a mathematical and graphical tool for general use. In this paper, the use of PNs is promoted as a modeling tool to organize the teaching of mathematical notions. The modeling presented in this work relies on the strong mathematical basis of PNs. As a case study, a model is constructed for teaching integrability of real functions, based on the evolution of the integral concept. With this aim, it is considered a real bounded function, defined on a closed and bounded interval, that is analyzed according to conditions which ensure or exclude its integration by Cauchy, by Riemann, by Lebesgue or by none of these integrals. The idea of modeling integrals using PN was originated in the context of mathematical classes, as the integral was being taught to first year engineering students.
\end{abstract}

Keywords: Modeling. Teaching. Petri Nets. Integral.

\title{
1 Introdução
}

O estudo de teorias, de métodos e de sistemas apropriados, que assegurem uma transmissão de conhecimentos de forma estruturada, é uma preocupação antiga das sociedades humanas. As escolhas educativas vão sendo influenciadas pelas religiões, pela política, pela economia, pelas ciências e pelas técnicas. A relação mestre/saber/aluno é, por sua vez, influenciada por essas escolhas.

Nas ciências matemáticas, em particular, o saber pode, ainda, sofrer influências da relação supracitada. Como exemplo, cita-se a construção do conceito de integral que está embasada em um forte formalismo matemático, o qual foi desenvolvido, em grande parte, devido à necessidade de se transmitirem, sem ambiguidades, os conteúdos a ele relacionados. De maneira mais geral, Dahan-Dalmedico e Peiffer (1986) afirmam que a obrigação de ensinar, imposta 
aos matemáticos do século XIX, foi uma das fontes do desenvolvimento do rigor em matemática.

Com o recente surgimento da Didática da Matemática (BROUSSEAU, 1986), propõem-se variadas abordagens de ensino das noções matemáticas, respeitando-se as características próprias dessa ciência e, ao mesmo tempo, os processos cognitivos do sujeito aprendiz. Nesta direção, são elaboradas engenharias didáticas (ARTIGUE, 1989), visando o ensino de conhecimentos específicos, e mapas conceituais (NOVAK; CAÑAS, 2007), relacionando de forma significativa diversos conteúdos, dentre outros exemplos.

Nesse mesmo contexto, sugere-se, neste trabalho, o uso de redes de Petri como uma ferramenta de modelagem voltada para o ensino de noções matemáticas. A modelagem apoia-se no fato de as redes de Petri terem, como característica fundamental, a representação de sistemas, utilizando como alicerce uma forte base matemática.

Como estudo de caso, apresenta-se uma modelagem para a organização do conteúdo de ensino sobre integrabilidade de funções reais baseada na evolução do conceito de integral, noção central do Cálculo Diferencial e Integral (CDI). Para isto, considera-se, inicialmente, uma função real, limitada e definida em um intervalo fechado e limitado da reta, que vai percorrendo uma rede de Petri e sendo analisada relativamente a condições suficientes que permitam a sua integrabilidade por Cauchy, por Riemann, por Lebesgue ou por nenhum destes. Em seguida, a função é integrada em uma sub-rede pelo processo mais adequado.

A ideia de se modelar esse conceito por redes de Petri originou-se no contexto da sala de aula, por ocasião de seu ensino aos alunos de primeiro ano do Curso de Graduação em Engenharia de Teleinformática (CGETI), da Universidade Federal do Ceará (UFC), na disciplina de Cálculo Fundamental.

Para situar este trabalho no universo das integrais, conta-se, a seguir, uma breve história desse conceito.

\section{Uma breve história do conceito de Integral}

Conceitos matemáticos podem surgir a partir da resolução de problemas rotineiros e bem contextualizados, ou a partir de situações de interesse apenas matemático. Dentre esses, alguns levaram milênios para ser elaborados, passaram por mentes brilhantes e continuam, até hoje, a sofrer transformações para atender às necessidades de uma comunidade científica. $\mathrm{O}$ conceito de integral, em particular, constitui-se em um exemplo que participou, e que ainda participa da 
resolução de problemas rotineiros, e que evoluiu, e continua evoluindo, para níveis de abstração ainda não alcançados pelas ciências aplicadas.

Pode-se considerar que o gérmen para a elaboração do conceito de integral tem suas raízes na necessidade primitiva de medir e de construir medidas.

Começando pela medida de um objeto geométrico bastante intuitivo, o segmento, a ideia de medir o seu comprimento parece natural. Contudo, ao se impor o fator precisão como condição, essa medida pode ser impossível.

Por outro lado, convencionando-se um tamanho como uma unidade de medida de comprimento, o problema de construir medidas de múltiplos fracionários da unidade tornou-se trivial após séculos de trabalho e de teoremas como o de Thales.

Durante muito tempo, não se cogitava sobre a existência de um comprimento que não fosse uma fração da unidade até que, por ironia do destino, o Teorema de Pitágoras apresentou uma medida que não era fracionária (SINGH, 1999). Mesmo assim, esta medida era construtível. E, como lidar com as medidas não construtíveis como $\sqrt[3]{2}$ ? Como aceitar as não-construtíveis, mas transcendentes como $\pi$ ?

Por outro lado, estimando-se que medir um comprimento é medir um intervalo da reta, sabe-se, hoje, que ao se considerar, por exemplo, o intervalo $[0,1]$ como unidade de medida e se estabelecer uma função para medir subconjuntos da reta, existem subconjuntos que não podem ser medidos por meio desta função. Cita-se, como exemplo, o conjunto de Vitali (ROYDEN, 1971).

Ao mesmo tempo, o homem precisava medir e construir medidas de porções do plano, do espaço etc. O que, na verdade, representa o outro lado da mesma medida de segmentos. De forma mais geral, na Geometria Euclidiana (sem o sistema de eixos coordenados), dado um objeto geométrico $A$, com certa medida, procura-se construir, apenas com régua e compasso, outro objeto geométrico $B$ que tenha a mesma medida de $A$. Provou-se (STEWART, 2004), porém, ser impossível tal construção quando grandezas não construtíveis estão implicadas nesse processo. Como exemplo, citam-se os famosos problemas gregos da quadratura do círculo e da duplicação do cubo.

Com a chegada da Geometria Analítica, alguns objetos geométricos passaram a ser representados por equações. Essa nova apresentação permitiu uma extensão do conceito de medida a uma classe maior de objetos.

No plano, se a região puder ser delimitada por curvas representadas por funções contínuas definidas em intervalos fechados, a integral de Cauchy (1789- 
1857) (e de vários outros matemáticos) representa uma maneira precisa de se medir tal região.

Ainda no século XIX, o matemático Riemann (1826-1866) (e outros, imprescindíveis) envolvido com a determinação da convergência de séries de Fourier, cujos coeficientes são dados por integrais de funções, desenvolveu uma teoria de integração caracterizada pelo estudo da convergência das somas superiores e das somas inferiores. Tal teoria, conhecida por integração de Riemann, permitiu a extensão do conceito de medida a porções menos comportadas do plano.

No início do século XX, Lebesgue provou que medidas de determinadas porções do plano pela Integral de Riemann são possíveis de ser realizadas se, e somente se, as funções envolvidas forem contínuas em quase toda parte (ou seja, descontínuas apenas em um subconjunto de medida nula no intervalo de definição da função) (RUDIN, 1976). Com o intuito de aplicar o conceito de integral a uma classe maior de funções, Lebesgue elaborou uma teoria de integração que é uma extensão da integral de Riemann. Para isso, ele considerou partições de um intervalo que contivesse o conjunto imagem da função em vez de partições do domínio, e estendeu a definição de medida de um intervalo para a medida de subconjuntos incomuns da reta.

A integral de Lebesgue, sendo mais geral, é mais complicada do que a de Riemann, entretanto, além de permitir a integração de uma classe maior de funções, alguns problemas relativos à convergência de sequências de funções integráveis podem ser resolvidos por meio da sua aplicação.

Noções mais gerais de integral foram introduzidas por Denjoy e Perron, em 1912 e em 1914, respectivamente, e por Henstock e Kurzweil, em meados do século vinte. Na perspectiva de generalizações, essa história não termina por aqui, segue inspirada pelo "pensamento matemático que pode ser caracterizado como uma aspiração ao completo" (BACHELARD, 1999, p. 36).

\section{Objetivos e motivação}

Essa história, contada em uma linguagem imprecisa e de maneira extremamente resumida, além disso, vista por um determinado ângulo, o das medidas, pode ser recontada de forma mais abrangente e completa usando linguagens formais.

Propõe-se, neste trabalho, a introdução de Redes de Petri como uma ferramenta de apoio à organização de conteúdos de ensino de noções matemáticas 
considerando-se suas características dinâmicas e a forma como conteúdos podem ser organizados em uma rede. Sugere-se, em particular, uma modelagem do conceito de integral usando Redes de Petri, na qual é notável a grande quantidade de informações interligadas, de maneira lógica e significativa, em um pequeno ambiente. Pretende-se, assim, recontar a história acima por meio de uma linguagem característica do campo das ciências matemáticas.

Contendo informações obtidas em textos diversos, interligadas em forma de proposições matemáticas por meio da linguagem das RPs, espera-se que o modelo ora proposto sirva ao professor tanto como fonte de referência quanto de organização de material de ensino a ser usado em sala de aula.

A motivação para se realizar uma modelagem do conceito de integral, e de estender esta ideia para outras noções matemáticas, surgiu em sala de aula, por meio de seu ensino na disciplina de Cálculo Fundamental, do primeiro ano do Curso de Graduação em Engenharia de Teleinformática (CGETI) da Universidade Federal do Ceará (UFC). Na ocasião, houve uma cobrança dos alunos por uma abordagem mais abrangente dessa noção relativamente aos conteúdos constantes nos livros de CDI mais adotados, tais como Leithold (1994), Finney, Wier e Giordano (2004), dentre outros. Para atender o interesse dos alunos, tornou-se imprescindível para o professor munir-se de um estudo mais rigoroso do conceito de integral, tanto pelo viés de uma epistemologia histórica, quanto por meio de uma fundamentação teórico-matemática mais formal. Visando à organização do ensino, grande parte deste estudo foi estruturada por meio das redes de Petri aqui apresentadas. De forma gradativa, ao longo de três anos, os conteúdos deste estudo foram sendo adequados ao nível de conhecimentos dos alunos de primeiro ano do CGETI. Uma apresentação mais completa deste trabalho de pesquisa pode ser lida em Barroso (2009).

Com esse objetivo, serão apresentadas nas seções seguintes: uma descrição das redes de Petri, do seu funcionamento e das suas principais propriedades; uma discussão sobre o uso de redes de Petri para a modelagem de processos matemáticos; uma proposta de modelagem da integrabilidade de funções reais por redes de Petri; uma comparação sucinta com a aplicação de mapas conceituais para o ensino, propostos por Novak e Cañas (2007); e, finalmente, as conclusões.

\section{Definição de Redes de Petri (RPs)}

Propostas por Carl Adam Petri (PETERSON, 1981), as redes de Petri 
(RP) constituem-se em uma ferramenta matemática e gráfica de uso geral. Elas permitem modelar o comportamento de sistemas dinâmicos a eventos discretos, descrever as relações existentes entre condições e eventos e visualizar propriedades tais como paralelismo, sincronização e compartilhamento de recursos.

Uma rede de Petri pode ser definida formalmente como uma quíntupla $\mathrm{RP}=\left(\mathrm{P}, \mathrm{T}, \mathrm{A}, \mathrm{W}, \mathrm{M}_{0}\right)$, em que:

- $\mathrm{P}=\left\{p_{1}, p_{2}, \ldots, p_{\mathrm{m}}\right\}$ é um conjunto finito de lugares;

- $\mathrm{T}=\left\{t_{1}, t_{2}, \ldots, t_{\mathrm{n}}\right\}$ é um conjunto finito de transições;

- $\mathrm{A} \subset(\mathrm{P} \times \mathrm{T}) \cup(\mathrm{T} \times \mathrm{P})$ é um conjunto finito de arcos;

- $\mathrm{W}: \mathrm{A} \rightarrow\{1,2, \ldots\}$ é a função peso associada aos arcos;

- $\mathrm{M}_{0}: \mathrm{P} \rightarrow\{0,1,2, \ldots\}$ é a marcação inicial.

As RPs são representadas graficamente por um grafo bi-partido, consistindo de dois conjuntos distintos de nós denominados lugares e transições e um conjunto de arcos com pesos associados, nos quais:

- Lugares são representados graficamente por um círculo;

- Transições são representadas graficamente por um seguimento de reta ou um retângulo;

- Arcos orientados e ponderados conectam lugares a transições e transições a lugares.

Uma RP modela os estados de um sistema e suas mudanças. Esses estados são modelados pela adição de fichas aos lugares da RP. A uma determinada distribuição de fichas, denomina-se marcação da RP. Na Figura 1, é apresentada uma RP especificando-se todos os seus elementos:

- $\mathrm{P}=\left\{p_{1}, p_{2}, p_{3}\right\}$

- $\mathrm{T}=\{a, b, c\}$;

- $A=\{(p 1, a),(a, p 2),(a, p 3),(p 2, b),(p 3, c),(b, p 1),(c, p 1)\}$;

- $\mathrm{W}=\{[(\mathrm{p} 1, \mathrm{a}), 1],[(\mathrm{a}, \mathrm{p} 2), 1],[(\mathrm{a}, \mathrm{p} 3), 1],[(\mathrm{p} 2, \mathrm{~b}), 1],[(\mathrm{p} 3, \mathrm{c}), 1],[(\mathrm{b}$, p1), 1], [(c, p1), 1]\};

- $\mathrm{M}_{0}=\{(\mathrm{p} 1,1),(\mathrm{p} 2,0),(\mathrm{p} 3,0)\}$.

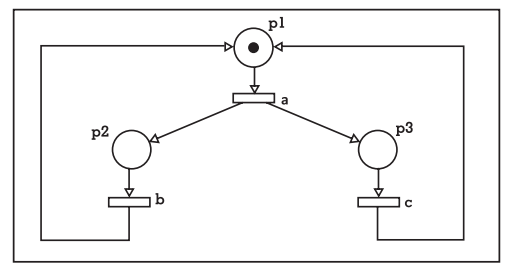

Figura 1 - Rede de Petri Ordinária com três lugares e três transições 
Os lugares conectados a uma transição no sentido lugar-transição são denominados de lugar de entrada daquela transição. Os lugares conectados a uma transição no sentido transição-lugar são denominados de lugares de saída daquela transição. Por exemplo, na RP apresentada na Figura 1, o lugar $p_{2}$ é lugar de saída e de entrada das transições $a$ e $b$, respectivamente.

A marcação de uma RP, em um dado instante, modela o estado da RP ou, mais precisamente, representa o estado do sistema modelado pela RP.

Em uma marcação, cada lugar contém um número inteiro (positivo ou nulo) de fichas. O número de fichas em um determinado lugar $p_{\mathrm{i}}$ será denotado por $M\left(p_{\mathrm{i}}\right)$ ou simplesmente $M_{\mathrm{i}}$.

A dinâmica de um sistema, ou suas mudanças de estado, é modelada em redes de Petri pela geração ou eliminação de fichas na rede.

Se a marcação define o estado da RP, a mudança de estado corresponde a uma evolução da marcação e essa evolução se produz pelo disparo das transições, o qual obedece à seguinte regra:

- Uma transição é dita habilitada ou sensibilizada se cada um de seus lugares de entrada contém um número de fichas maior ou igual ao peso do arco que o conecta à transição.

- Uma transição habilitada pode ou não disparar.

- Ao disparar, fichas são removidas de cada lugar de entrada da transição disparada e fichas são acrescentadas aos seus lugares de saída. A quantidade de fichas removidas e acrescentadas depende do peso do arco.

Observa-se que, na Figura 1, a transição $a$ está habilitada, pois $p_{1}$ é o único lugar de entrada de $a$, existe uma ficha em $p_{1}$ e o peso do arco que liga $p_{1}$ a $a$ é igual a 1. Na Figura 2, é apresentada a nova marcação da rede após o disparo de $a$. Nesse caso, uma ficha foi retirada de $p_{1}$, uma ficha foi acrescentada a $p_{2}$ e uma ficha foi acrescentada a $p_{3}$, pois é esse o peso dos arcos que ligam, respectivamente $a$ a $p_{2}$ e a $p_{3}$.

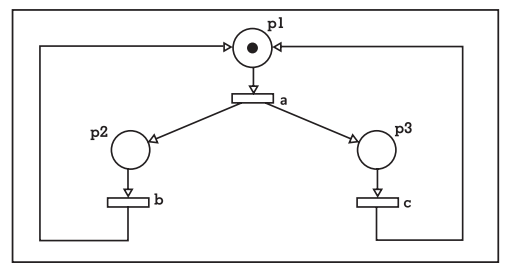

Figura 2 - Rede de Petri Ordinária da Figura 1 após disparo da transição $a$

Como pode ser visto pela Figura 2, uma nova marcação resultante do 
disparo de uma transição representa o novo estado do sistema modelado. Vale lembrar que o disparo de uma transição é uma ação atômica (disparo instantâneo). A nova marcação da rede é $\mathrm{M}_{1}=\{(\mathrm{p} 1,0),(\mathrm{p} 2,1),(\mathrm{p} 3,1)\}$ ou, simplesmente, $\mathrm{M}_{1}$ $=\left(\begin{array}{lll}0 & 1 & 1\end{array}\right)$.

As RPs oferecem ferramentas de análise que podem revelar informações importantes sobre a estrutura e o seu comportamento dinâmico do sistema modelado por ela. Entretanto, devido ao baixo poder de representação de uma ficha, a modelagem de sistemas complexos pode gerar diagramas grandes e de difícil leitura. Para lidar com esse tipo de restrição, conceberam-se as RPs de Alto Nível (RPA), que tornam os modelos menores e de mais fácil visualização e análise em relação às RPs. Para isso, as fichas armazenadas em lugares recebem um tipo de dado (cor), podendo representar estruturas mais complexas, como listas (estruturas de dados com múltiplos elementos) e registros (elementos de dados compostos por outros elementos). Uma linguagem de programação é associada à rede, permitindo customizar o controle dos disparos das transições, realizar operações nas informações das fichas removidas e atuar na geração de informações dos lugares de entrada. Também podem ser construídas subredes, formando modelos hierárquicos, potencializando a segmentação e contextualização de partes da estrutura global da rede.

Dentre as redes de alto nível, destacam-se as redes de Petri coloridas (RPC), que são atualmente as mais usadas no mundo e foram, por isso, empregadas no presente trabalho (JENSEN, 1997).

As redes de Petri coloridas (RPC) são formadas por uma 9-tupla que pode ser representada por $\mathrm{RPC}=(\mathrm{P}, \mathrm{T}, \mathrm{A}, \Sigma, \mathrm{V}, \mathrm{C}, \mathrm{G}, \mathrm{E}, \mathrm{I})$, em que:

- $\mathrm{P}$ é um conjunto finito de lugares;

- T é um conjunto finito de transições;

- A é um conjunto de arcos direcionados ligando lugares a transições e transições a lugares;

- $\Sigma$ é um conjunto finito de conjuntos de cores não vazio;

- V é um conjunto finito de variáveis do tipo $\Sigma$;

- C é a função que atribui um conjunto de cores a cada lugar da rede;

- G é a função que atribui uma guarda a cada transição da rede;

- E é a função que atribui uma expressão a cada arco da rede;

- I é a função de inicialização que atribui uma marcação inicial aos lugares.

Neste trabalho é utilizada uma ferramenta automatizada, para modelagem e análise de RPC, denominada CPNTools ${ }^{l}$, que é gratuita para instituições educacionais e de pesquisa.

${ }^{1}$ Disponível em: http://wiki.daimi.au.dk/cpntools/_home.wiki 


\section{Uso de Redes de Petri para modelagem de processos matemáticos}

As RPs, embora criadas com o objetivo de modelar sistemas a eventos discretos, podem ser usadas para modelar sequências de ações ou atividades de qualquer natureza. Como exemplo, cita-se o emprego de RPs como ferramenta de apoio à modelagem de processos de negócios, mais precisamente, como tecnologia de gerenciamento workflow (PÁDUA et al., 2004; AALST, 1998). Outros exemplos dizem respeito à sua utilização como ferramenta de modelagem de processos ligados ao ensino e à aprendizagem (PARK; KIM, 2008).

No âmbito deste trabalho, a motivação de modelar por redes de Petri reside, originalmente, no fato de ser a matemática, segundo Russel (2007), uma ciência que se confunde com a lógica. Nesse sentido, sendo os eventos relacionados em uma RP de maneira lógica, as suas propriedades podem ser satisfatoriamente aproveitadas para a representação de conceitos e de processos matemáticos.

Relativamente ao modelo aqui proposto, as fichas, definidas na seção anterior, contêm estruturas que armazenam os atributos significativos, selecionados para o problema em estudo. Várias cores podem ser adotadas para os lugares da rede ou pode-se trabalhar com apenas uma delas, como será apresentado na rede construída para a análise da integrabilidade. A convenção para a representação da ficha deve ser especificada de maneira não ambígua, indicando o significado de cada campo da estrutura de dados utilizada.

As transições representam as operações, que podem ser definidas em um alto nível de abstração, quando, em geral, podem ser desdobradas em subredes, detalhando uma operação contextualizada dentro de um subprocesso.

É importante notar que não se trata de um tutorial para a resolução de um problema e nem do desenvolvimento de sua solução em si. O objetivo é oferecer um suporte estrutural no qual, de maneira especial, os professores e, eventualmente, os alunos, possam visualizar, em diversos níveis de abstração, devido à hierarquização da rede, os conceitos necessários para o desenvolvimento de seu planejamento didático.

Remetendo ao planejamento didático e à elaboração do material de ensino, e tendo em perspectiva a teoria proposta por Ausubel (MOREIRA,1999), uma das condições para a ocorrência de aprendizado é que ele seja organizado de forma potencialmente significativa para o aluno. De acordo com Pontes e Silva (2006), as novas ideias devem ser expostas de maneira não arbitrária e de tal forma que a sua natureza possua significação lógica, relacionando-as a ideias 
que estejam dentro do domínio da capacidade humana de aprendizagem. Nesse caso, o conteúdo e a organização do material a ensinar devem ser vinculados aos conhecimentos prévios dos alunos aos quais ele se dirige.

Com o objetivo de ressaltar essa condição para a aprendizagem, no modelo proposto os conhecimentos necessários à execução de uma operação são representados por quadrados coloridos, em forma de legenda, nas proximidades da transição que representa essa operação (ver Figura 5). As informações associadas às legendas podem servir de orientação ao professor sobre quais conhecimentos prévios deve ter o aluno para compreender o que vai ser ensinado.

Na próxima seção, propõe-se um modelo em Redes de Petri Coloridas para analisar a integrabilidade de funções reais. Representam-se, de maneira lógica e significativa, diversas conexões entre as integrais de Cauchy, de Riemann e de Lebesgue. Normalmente, essas integrais são ensinadas separadamente, em disciplinas distintas, e nessa ordem. O interesse, aqui, reside em caracterizar a noção de integrabilidade pela perspectiva da generalização, dificilmente alcançada por meio de um ensino fragmentado.

\section{Representação da análise da integrabilidade por Redes de Petri coloridas}

Nesta seção, apresenta-se uma conexão de um conjunto de informações dispersas em vários textos de livros ou artigos concernentes à construção do conceito de integral. A formação deste conceito é considerada, aqui, pelo viés da análise da integrabilidade das funções reais relativamente às integrais de Cauchy, de Riemann e de Lebesgue.

Observa-se que um primeiro curso de integral deixa no aluno, em geral, a certeza de que todas as funções são não apenas integráveis à Riemann, mas têm primitivas. Logo, assumindo o papel de um aluno de primeiro ano, analisar a integrabilidade de uma função parece desnecessário. Isto ocorre porque, de um modo geral, a representação que se tem de uma função é a de que ela é não apenas contínua, mas suave em todos os pontos, portanto, integrável. Porém, no universo das funções reais, a quantidade de funções suaves é muito inferior à de funções descontínuas em todos os pontos de seu domínio (CHEN; SMITH, 2008), as quais, neste caso, não são integráveis à Riemann. Esse fato, contrário à intuição, já justifica a necessidade de se fazer tal análise.

Na modelagem da integrabilidade ora proposta, parte-se de uma função 
$f$ real e limitada, definida em um intervalo fechado e limitado da reta, ou seja:

$f \in F$, em que $F=\{g:[a, b] \rightarrow R ; g$ é limitada $\}$

Em princípio, não é feita nenhuma outra restrição sobre $f$. Pretende-se integrar $f$, caso seja possível, por Cauchy, por Riemann ou por Lebesgue.

Para isso, à medida que $f$ vai percorrendo a rede, uma análise de suas propriedades, relativas a sua continuidade, é realizada para indicar o melhor caminho a seguir.

Um modelo hierárquico foi construído para representar o conjunto de etapas constituintes do processo de análise da integrabilidade de uma função $f$ real. A Figura 3 apresenta de maneira esquemática a hierarquia das subredes que compõem o modelo.

Na RP, são considerados, portanto, três processos de integração de uma função: integração por Cauchy, por Riemann ou por Lebesgue.

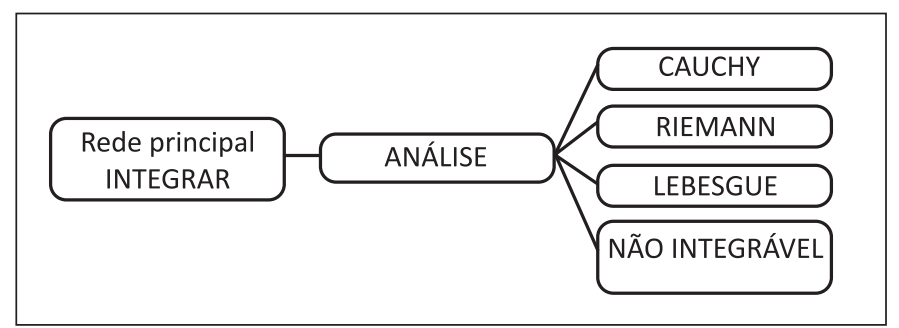

Figura 3 - Hierarquia das Subredes representativas do Processo de Análise da Integrabilidade

A Rede Principal é apresentada na Figura 4, em que se pode ter uma idéia geral dos passos realizados da análise à resolução da Integral de uma função $f$.

$\mathrm{Na}$ RP da Figura 4, ao passar pela transição Analisar, a função é submetida a uma análise de propriedades que vão de condições mais restritivas, como a continuidade, a condições mais gerais, como a mensurabilidade. São condições que permitem a integração por Cauchy (C), por Riemann (R), por Lebesgue (L) ou por nenhum deles. Nesse processo, a noção de integral vai se estendendo a uma definição mais geral que, ao mesmo tempo, exige maior abstração. 


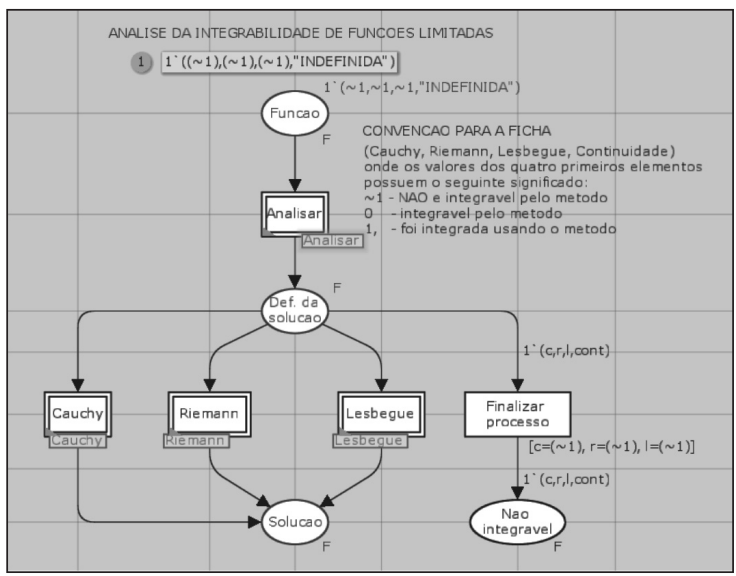

Figura 4 - Rede Principal apresentando a hierarquia que contém a análise da integrabilidade e o cálculo das integrais

Todas as transições da Rede principal apresentada na Figura 4, exceto a transição nomeada Finalizar processo, são chamadas de transições de substituição. Representada por um retângulo de moldura dupla, uma transição de substituição indica que o processo a ela associado é modelado em uma subrede, portanto, representado independentemente. No modelo proposto, as quatro subredes são modeladas para descrever as etapas necessárias para: verificar a integrabilidade da função (transição Analisar), integrar $f$ por Cauchy, integrar $f$ por Riemann ou integrar $f$ por Lesbegue.

A subrede apresentada na Figura 5, relativa à transição de substituição Analisar da Figura 4, representa a execução da análise da função $f$.

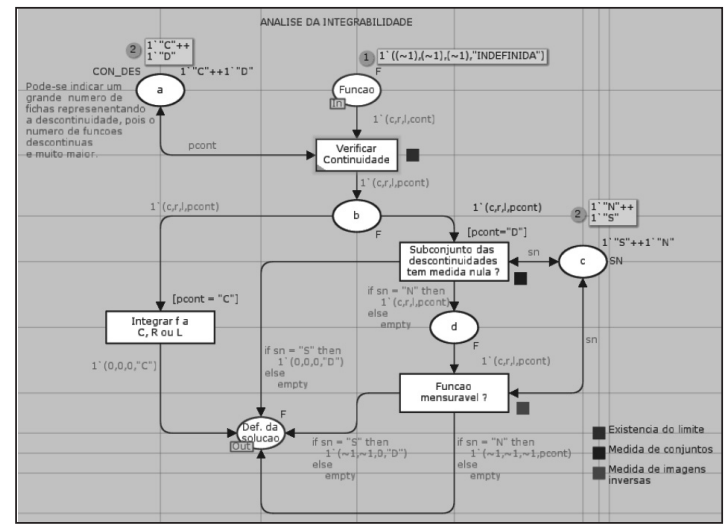

Figura 5 - Sub-rede apresentando os passos para a análise da integrabilidade 
Do conjunto $F$, retira-se, de maneira aleatória, uma função $f$, representando a escolha arbitrária de uma função num conjunto infinito de possibilidades. Na abstração aqui utilizada, essa função visita vários lugares da rede que são interligados por caminhos, em que cada caminho é representado por uma transição. Em cada transição à qual $f$ é submetida, realiza-se a análise de alguma condição que indicará qual ou quais os lugares ela deve visitar para que possa ser integrada.

A ficha que representa a função $f$ que percorre a rede possui uma estrutura composta de quatro campos: três campos de valor inteiro e uma string de caracteres. Os três primeiros campos representam, respectivamente, a possibilidade de a função ser integrada por Cauchy, Riemann e Lesbegue. Para isso, convencionou-se o seguinte significado para os valores desses campos:

-( 1) - A função ainda não foi analisada para esse método.

-(0) - A função foi analisada para esse método, podendo ser integrada por ele.

-(1) - A função foi analisada para esse método e verificou-se que não é possível ser integrada por ele.

O quarto e último campo, representativo da função $f$ em análise, indica se a mesma é contínua ou não, podendo assumir os valores indefinida, se ainda não foi verificada, C, quando se trata de uma função contínua, ou D, caso seja uma função descontínua.

Enfatiza-se que, no lugar inicial, chamado de Função na rede da Figura 4, a única condição imposta à função $f$ é que ela seja limitada e definida em um intervalo fechado e limitado da reta.

Ao sair desse lugar, $f$ segue um caminho que a leva a uma subrede na qual é feita uma análise da sua continuidade. Usando a extensão proposta para representar os conhecimentos envolvidos em cada parte do processo, o ícone (quadrado vermelho) na transição Verificar Continuidade indica, conforme o texto associado na legenda, a necessidade de conhecimentos sobre existência do limite. Verificada a continuidade $\operatorname{de} f$ :

- Caso $f$ seja contínua, ela passa por uma transição que informa que ela pode ser integrada por $\mathrm{C}, \mathrm{R}$ ou $\mathrm{L}$, indicando Cauchy, Riemann e Lesbegue. Escolhe-se um desses métodos, integra-se $f$ e o processo termina para essa função e podemos subir na hierarquia da rede, retornando à rede ilustrada na Figura 4.

- Caso contrário, $f$ passa por uma transição que faz uma análise do seu tipo de descontinuidade (Subconjunto das descontinuidades tem 
medida nula?). Para isso, o ícone azul indica a necessidade de conhecimentos sobre medidas de subconjuntos da reta. A saída dessa transição é condicional, como mostram as instruções escritas nos seus arcos de saída. Tais condições verificam:

- Se $f$ for contínua em quase todo ponto (qtp), quer dizer, se o conjunto de seus pontos de descontinuidade tem medida nula, ela pode ser integrada por R ou L. Nesse caso, remonta-se ao nível superior da rede, onde escolhe-se o método, integra-se a função e o processo termina.

- Caso contrário, se o conjunto dos pontos de descontinuidade tem medida não nula, ela vai para o lugar d guardando essa informação. Na subrede da Figura 5, essa condição é simulada aleatoriamente pela retirada de uma das fichas do lugar c. Do lugar d, $f$ percorre uma transição que faz uma análise da sua mensurabilidade. Para essa verificação, é necessário o conhecimento sobre medida de imagens inversas de intervalos, como indica o ícone verde associado à transição Função Mensurável?. A análise para essa transição procede da seguinte maneira:

a) Sendo f mensurável, garante-se a sua integrabilidade por L e, novamente, remonta-se ao nível superior da hierarquia, integra-se a função e acaba o processo.

b) Caso contrário, f não pode ser integrada por C, por R e nem por L. Tais informações são configuradas na ficha e, no nível superior da hierarquia da rede, finaliza-se o processo para $f$.

Note-se que na RP da Figura 5 está estruturada a forma como o conceito de integral foi evoluindo a partir de noções mais básicas (porém, não tão simples), como a de continuidade, a noções mais gerais, como a de mensurabilidade. Além disso, é possível extrair da rede alguns resultados importantes como:

Se $F$ for contínua, então f é integrável a C, R e L.

Se $F$ não é contínua qtp, mas é mensurável, então $f$ é integrável à Lebesgue.

Desses dois resultados, pode-se questionar, por exemplo, sobre a veracidade de suas recíprocas ou, ainda, se funções contínuas são necessariamente mensuráveis. E, assim, grande parte da teoria vai sendo construída à medida que se transita pela RP.

Representa-se, na Figura 6, a subrede na qual se processa uma integração de uma função usando Cauchy. Optou-se por apresentar, aqui, apenas 
esse procedimento, e não R ou L, por ser a integral de Cauchy a mais conhecida. Ela é, em geral, a única integral a ser, pelo menos parcialmente, abordada em uma primeira disciplina de Cálculo Diferencial e Integral (BARROSO et al., 2007).

Ressalta-se que na construção da integral denominada, nesse artigo, de Cauchy, o cálculo das somas superiores e das somas inferiores está condicionado à existência do valor máximo absoluto e do valor mínimo absoluto em qualquer subintervalo do intervalo de integração. Observa-se que não só as funções contínuas atendem a essa condição. Entretanto, no contexto de uma disciplina de cálculo que trata da integral de funções contínuas, o Teorema do Valor Extremo garante a existência desses extremos absolutos.

Destaca-se que, embora haja uma grande concentração de informações nas redes expostas, a modelagem ora apresentada pode ser vista como uma representação sintética, mas sem ambiguidades, de um estudo históricoepistemológico-científico sobre o conceito de integral o qual foi realizado para dar suporte matemático a esta pesquisa.

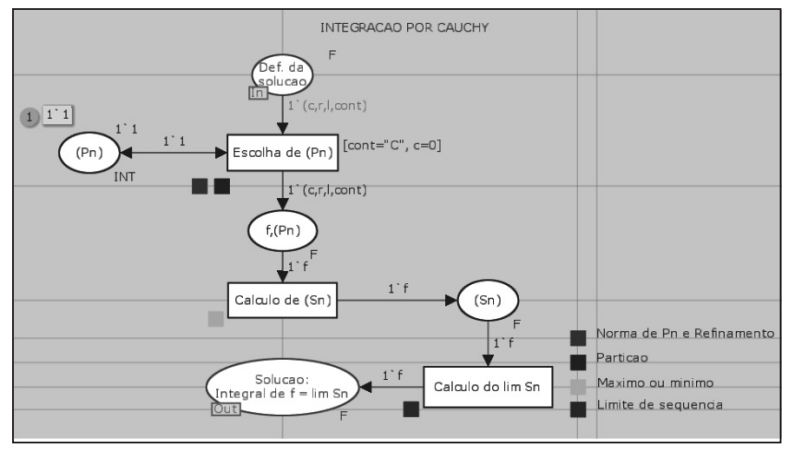

Figura 6 - Rede representativa da Resolução por Cauchy

\section{Por que não foram usados mapas conceituais?}

A linguagem proposta nesta pesquisa para o uso de Redes de Petri em contextos educacionais lembra, devido as suas características gráficas e esquemáticas, os Mapas Conceituais (MCs), outra ferramenta bastante usada no meio educacional para representar conceitos (não apenas matemáticos) e suas associações. Faz-se necessário, portanto, uma rápida comparação entre RPs e MCs.

Os MCs são apropriados para organizar e representar conhecimentos 
através de uma linguagem simples (NOVAK; CAÑAS, 2007). Em um mapa conceitual, os conceitos são colocados em elipses ou retângulos que, quando relacionados entre si, são ligados por um segmento. Quando necessário, podese descrever textualmente essa relação.

Diferentemente dos Mapas Conceituais, uma RP pode ser usada para modelar sistemas dinâmicos, percebendo-se de maneira não ambígua as pré e pós-condições associadas a uma transição. A cada momento, as fichas depositadas nos lugares da rede determinam o estado do sistema, o que não é representável usando mapas conceituais. Em um mapa conceitual as conexões não indicam caminhos, mas associações lógicas entre conceitos, que podem ser representadas diferentemente para cada indivíduo em função da sua estrutura cognitiva.

Portanto, a organização de certas noções por meio de RP se torna interessante quando as propriedades da RP, tais como conflito, tomada de decisão, dinâmica, podem ser identificadas com o processo de construção do conteúdo envolvido.

Enquanto os mapas conceituais objetivam representar a estrutura cognitiva do indivíduo e a maneira como o mesmo inter-relaciona os conceitos adquiridos, as RPs, da forma como se propõe neste trabalho, fazem a abstração das operações necessárias aos processos de construção ou de resolução de problemas matemáticos, evidenciando os momentos de aplicação dessas operações e, eventualmente, a presença de abordagens alternativas ou a existência de subproblemas. Para cada operação, registram-se os requisitos, em termos de conhecimentos, necessários à sua realização. Não se trata, portanto, de um modelo da estrutura cognitiva de aprendizes, embora possa ser utilizado como organizadores prévios para facilitar a associação lógica entre conteúdos.

Deve-se deixar claro que esta breve comparação não tem por intuito minimizar a importância do uso de mapas conceituais no ensino/aprendizado, mas de oferecer ao professor outra alternativa de ferramenta de apoio que, em determinados contextos, pode levar a resultados mais eficazes.

\section{Conclusões}

Segundo D'Amore (2005), um conceito está permanentemente em fase de construção e, nessa construção, encontra-se a parte mais problemática e mais rica de seu significado. No contexto da sala de aula, elaborar a construção 
de uma noção não trivial quanto à de integral não se constitui, portanto, em uma tarefa fácil. Entretanto, o domínio do conteúdo por parte do professor corresponde a um dos pressupostos para que essa tarefa seja bem cumprida.

De acordo com Pais (2001), quando um sujeito passa a ter domínio sobre um determinado saber, é possível desencadear uma ação mais transformadora, geradora de novos saberes. Nesse sentido, propor-se a utilização de RP para a organização do conteúdo de ensino de determinadas noções matemáticas, como o caso da integrabilidade de funções reais, pode trazer relevantes contribuições na medida em que:

- Com abstrações gráficas construídas com a linguagem das RPs é possível apresentar um conjunto de informações essenciais e representar uma interconexão lógica entre uma grande quantidade de conteúdos que não são encontrados, normalmente, em um único livro. Para a modelagem das RPs aqui apresentadas, por exemplo, foram feitas pesquisas em livros de CDI, de análise real, de teoria da medida, de história da matemática, dentre outros. Esse tipo de representação pode ser utilizado por professores como instrumento de apoio para a organização e contextualização do conteúdo de suas aulas. Não se descarta, ainda, a possibilidade de essa representação gráfica, em maior nível de abstração, ser utilizada como suporte significativo para que os próprios alunos estabeleçam uma melhor relação entre conteúdos de diferentes disciplinas.

- Observa-se também, nas redes acima, que a organização dos conteúdos carrega em si uma forma de raciocinar sobre os objetos matemáticos que é característica ao campo de conhecimentos da matemática. Percorrendo, por exemplo, a RP da Figura 5, podem-se enunciar os seguintes teoremas avançados da teoria de integração:

Se $f:[a, b] \rightarrow R$ é limitada e mensurável então $f$ é integrável à Lebesgue.

Se $f:[a, b] \rightarrow R$ é uma função limitada e contínua qtp então $f$ é mensurável.

Proposições desse nível, fundamentadas em teorias matemáticas, podem orientar o professor tanto na concepção de suas sequências de ensino, quanto na escolha de atividades que conduzam o aluno a alcançar um nível maior de generalidade.

Com o intuito de contribuir, de maneira mais geral, com uma sistemática que auxilie o professor na organização de conteúdos complexos, tais como os 
conceitos de cálculo, interligando-os de forma matematicamente coerente e tornando-os mais acessíveis ao professor, propõe-se a introdução de Redes de Petri como uma ferramenta de auxílio na organização de material de ensino, considerando-se suas características dinâmicas e a forma como conteúdos podem ser organizados em uma rede. Todavia, é provável que, sozinho, o professor de matemática não consiga realizar todos os passos para a sua implementação, pois, para se elaborar a modelagem deste trabalho, contou-se com a participação de um profissional de informática, de um matemático, de um especialista em RP, de um profissional de Educação Matemática e, para dar significado à pesquisa, de um professor atuando em sala de aula.

O modelo aqui apresentado refere-se a conteúdos matemáticos. Porém, sendo esta pesquisa realizada no âmbito da engenharia e sendo ferramentas de modelagem, tais como as redes de Petri, bastante familiares aos engenheiros, a sua utilização pelo professor engenheiro para a organização de seu material de ensino em disciplinas específicas da engenharia seria de mais fácil implementação e também ensejaria um aporte significativo no caso de conteúdos que, da mesma forma que em matemática, pudessem se beneficiar das propriedades das RPs.

\section{Referências}

AALST, W. The application of Petri nets to workflow management. The Journal of Circuits, Systems and Computers, London, v. 8, n. 1, p. 1-53, 1998.

ARTIGUE, M. Ingénierie Didactique. Recherches en Didactique des Mathématiques, Paris, v. 9, n. 3, p. 281-308, 1989.

BACHELARD, G. Le nouvel sprit scientifique. 6. ed. Paris: Quadrige/PUF, 1999.

BARROSO, N. Um Modelo de Ensino dos Conceitos de Cálculo para os Cursos de Engenharia Fundamentado em uma Epistemologia Histórica e Baseado na

Metodologia da Engenharia Didática: validação por meio do Conceito de Integral. 2009. 187f. Tese (Doutorado em Engenharia de Teleinformática) - Universidade Federal do Ceará, Fortaleza, 2009.

BARROSO, N.; SOARES, J. M.; MOTA, J. C. M; NETO, H. B. Uma Sequiência de Ensino para a Introdução do Conceito de Integral de Riemann. In: ENCONTRO NACIONAL DE EDUCAÇÃO MATEMÁTICA, 9., 2007, Belo Horizonte. Anais... Belo Horizonte: Sociedade Brasileira de Educação Matemática (SBEM), 2007, p. 1-17.

Disponível em: $<$ http://www.sbem.com.br/files/ix_enem/Html/ comunicacaoCientifica.html>. Acesso em: 21 mar. 2013. 
BROUSSEAU, G. Fondements et méthodes de la Didactique des Mathématiques. Recherches en Didactique des Matematiques, Paris, v. 7, n. 2, p. 33-116, 1986.

CHEN, J.; SMITH, S. Cardinality of the Set of Real Functions with a Given Continuity Set. Pi Mu Epsilon Journal, Worcester, Massachusetts, v. 12, n. 8, p. 449-454, 2008. Disponível em: <http://www.sju.edu/ smith/Current_Courses/continuitysetsrev.pdf>. Acesso em: 21 mar. 2013.

DAHAN-DALMEDICO, A.; PEIFFER, J. Une Histoire des Mathématiques: Routes et Dédales. Paris: Le Seuil, 1986.

D’AMORE, B. Epistemologia e didática da Matemática. São Paulo: Escrituras, 2005.

FINNEY, R.; WEIR, M.; GIORDANO, F. Cálculo George B. Thomas. 10. ed. São Paulo: Pearson-Addison Wesley, 2004. (Volume 1).

JENSEN, K. Coloured Petri Nets. Basic Concepts, Analysis Methods and Practical Use. 2. ed. Berlin: Springer-Verlag, 1997. (Volume 1).

LEITHOLD, L. Cálculo com geometria analítica. 3. ed. São Paulo: Harbra, 1994.

MOREIRA, M. Teorias da Aprendizagem. São Paulo: EPU, 1999.

NOVAK, J. D.; CAÑAS, A. J. Theoretical Origins of Concept Maps, How to Contruct Them, and Uses in Education. Refleting Education, Londres, v. 3, n. 1, p. 29-42, Nov. 2007.

PÁDUA, S.; SILVA, A.; PORTO, A.; INAMASU, R. O potencial das redes de Petri em modelagem e análise de processos de negócios. Gestão \& Produção, São Carlos, v. 11, n. 1, p. 109-119, jan./abr. 2004.

PAIS, L. C. Didática da Matemática: uma análise da influência francesa. 2. ed. Belo Horizonte: Autêntica, 2001. (Coleção Tendências em Educação Matemática).

PARK, S.; KIM, Y. Applying Petri Nets to Model Customized Learning and Cooperative Learning with Competence. International Journal of Computer Science and Network Security, Seul, v. 8, n. 2, p. 127-132, Feb. 2008.

PETERSON, J. L. Petri Net Theory and the Modeling of Systems. New Jersey: Prentice Hall, 1981.

PONTES, J.; SILVA, J. Teoria da Aprendizagem Significativa de David Ausubel: Perguntas e Respostas. Série-Estudos: Periódicos do Mestrado em Educação da UCDB, Campo Grande, n. 21, p. 117-130, jan./jun. 2006. 
ROYDEN, H. Real Analysis. 3. ed. New Jersey: Prentice Hall, 1971.

RUDIN, W. Principles of Mathematical Analysis. 3.ed. New York: McGraw-Hill, 1976.

RUSSEL, B. Introdução à Filosofia Matemática. 1. ed. Rio de Janeiro: Zahar, 2007.

SINGH, S. O Último Teorema de Fermat. 5. ed. Rio de Janeiro: Record, 1999.

STEWART, I. Galois Theory. 3. ed. Flórida: Chapman \& Hall/CRC, 2004.

Submetido em Dezembro de 2011. Aprovado em Maio de 2012. 